\title{
PAPER
}

\section{High-order statistic for mean scatterer spacing estimation for human tissues characterization}

\author{
Abdelhafid Kaddour ${ }^{1, *}$, Bachir Bouhadef ${ }^{1, \dagger}$, \\ Mohamed Faouzi Belbachir ${ }^{1}$ and Jean Michel Rouvaen ${ }^{2}$ \\ ${ }^{1}$ University of Science and Technology of Oran Mohamed Boudiaf (USTO-MB), \\ City, Oran 31000, Algeria \\ ${ }^{2}$ IEMN (UMR 8520 CNRS), Departement OAE, Universite de Valenciennes, \\ 59313 Valenciennes Cedex, France
}

(Received 13 October 2009, Accepted for publication 16 June 2010)

\begin{abstract}
The mean scatterer spacing (MSS) has been identified as an important parameter for characterizing human tissues presenting a semi-regular lattice texture. Most of the work in this area involves the uses of an estimated power spectrum density (PSD) of an autoregressive (AR) model to deduce the MSS from backscattered ultrasonic signals. In this paper, we propose a new method of MSS estimation using the third and fourth-order cumulants, named here TFOCs, of the AR parametric model. The results obtained by this new method is compared with those obtained using PSD.
\end{abstract}

Keywords: Tissue characterization, Ultrasound backscattered signal, Signal processing, Cumulants

PACS number: 43.80.Jz, 43.80.Ev, 43.60.Uv, 43.60.Pt [doi:10.1250/ast.32.1]

\section{INTRODUCTION}

Ultrasonic imaging has been recognized as an invaluable tool in medical diagnosis. Ultrasonic tissue characterization techniques offer the promise of noninvasive discrimination between normal and diseased tissues. Ultrasound B-Scan imaging comprises in the observation of signals (echoes) returned from scatterers located inside the tissue under investigation and gives rise to so-called speckle $[1,2]$. The clinical imaging interpretation is still limited and based on the radiologist's evaluation.

The aim of actual ultrasonic research is to overcome these limits using signal processing methods. Hence various attempts have been made for tissue characterization using A-Scan. Many works have already been reported, providing quantitative information useful in tissue characterization by exploiting the backscattered radio frequency (RF) signal [3-5]. Useful parameters can be extracted from the processed ultrasonic backscattered signal, such as the ultrasonic transducer's impulse response, integrated backscatter, attenuation, reflection coefficients, celerity, mean scatterer spacing (MSS) and density of scatterers [6-12].

\footnotetext{
*e-mail: kaddour@univ-usto.dz

†e-mail: bouhadef@gmail.com
}

The MSS parameter, which is our interest, might carry information about the tissue state, and can be useful for classifying some biological tissues. Several authors supposed that ultrasonic scattering from human tissues, such as liver, has two principal components. The first one, which is highly coherent, is due to a semiregular structure, formed probably by lobules or portal triads, named scatterers. The second one, which is highly incoherent, corresponds to a random (irregular) structure that consists of diffuse scatterers [13].

Hence, the MSS of the semiregular scatterers can be determined from the information carried by the magnitude and phase spectrum of backscattered ultrasonic signals. If we blindly use the backscattered signal, the estimated mean scatter spacing from healthy tissues would be found to be $1-1.5 \mathrm{~mm}$, and for abnormal tissues, it would be slightly greater [14]. However, various methods, based on the power spectrum density (PSD) or autocorrelation of the magnitude of the spectrum $[15,16]$, start with the assumptions of Gaussianity and a minimum phase [17-20]. In other words, all these methods depend on the second-order spectrum or second-order statistics. In order to overcome these limits, the third and fourth-order cumulants (TFOC) [21] of an autoregressive (AR) model are used here for the estimation of MSS, and this parameter is compared with that determined using the PSD of an AR model (PSD- 
AR) [18]. Furthermore, we assume, in this work, that the speckle distribution follows a Rayleigh law. The high-order statistics have already been used for tissue response modeling based on Ultrasonic B-scan images by Abeyrantne et al. [19].

In this present work, a liver tissue simulation model is first proposed and defined in the first section. The second section is dedicated to the theoretical presentation of PSD and TFOC methods. Then, the MSS is determined using simulation. Finally, comparisons between results obtained using both above-mentioned methods (PSD and TFOC) are given, showing their advantages and disadvantages.

\section{THE SIMULATION MODEL}

The impulse response of tissue structure is modelled as [13]

$$
g(t)=g_{\mathrm{r}}(t)+g_{\text {ir }}(t),
$$

where

$$
g_{\mathrm{r}}(t)=\sum_{i=1}^{L} a_{i} \delta\left(t-\tau_{1, i}\right)
$$

and

$$
g_{\text {ir }}(t)=\sum_{i=1}^{M} b_{i} \delta\left(t-\tau_{2, i}\right) .
$$

The first and second terms, of the right part of Eq. (1) represent, respectively, the regular structure (pseudoperiodic, resolvable) $g_{\mathrm{r}}(t)$ that characterizes the tissue and the irregular structure (unresolvable, diffuse) $g_{\text {ir }}(t)$ due to individual liver cells and other unresolvable, random tissue microstructures (speckles). The coefficients $a_{i}$ and $b_{i}$ are the relative strengths of semiregular and irregular scatterers (which include all physical phenomena such as attenuation, diffusion section, etc.), $\delta(\cdot)$ is the Dirac function, $L$ is the number of semiregular scatterers, $M$ is the number of irregular scatterers, and $\tau_{1, i}$ and $\tau_{2, i}$ are the time delays from, respectively, the semiregular and irregular scatterers to the receiver. The relative strengths $a_{i}$ and $b_{i}$ will be considered as two independent random variables uniformly distributed in interval $[0,1]$.

The amount of time separating two adjacent semiregular scatterers is a random variable with a Gamma distribution according to Landini and Verrazzani [4], while Simon et al. [7] proposed a Poisson distribution for the time separating two adjacent semiregular scatterers. The Poisson distribution is used to model events occurring in a fixed period of time. In our case, the presence of a semiregular scatterer at a specific distance (or time) is considered as an event. If we wish to estimate the number of scatterers from the time interval (or space interval), the semiregular scatterer spacing can be modelled by a temporal Poisson distribution:

$$
p\left(x=k, \lambda_{1}\right)=\exp \left(-\lambda_{1}\right) \frac{\lambda_{1}^{k}}{k !}, \quad k=0,1,2, \ldots
$$

where $\lambda_{1}$ is the parameter of this distribution, and $x$ is the number of scatterers in the time interval. To convert this number to a time distribution, we divide this number by $\lambda_{1}$ (to normalize it) and multiply it by $\mu$, which is the MSS in the time domain. Hence, the time interval $\Delta \tau_{1}=\tau_{1, i+1}-$ $\tau_{1, i}$ between two adjacent semiregular scatterers is distributed according to $x \mu / \lambda_{1}$ with mean $\mu$ and variance $\sigma^{2}=\sigma_{x}^{2} \lambda_{1}^{2}$. For the irregular scatterers model, Wagner et al. [1] have shown that when the number of those scatterers per resolution cell of the transducer is sufficiently large, diffuse scattering having Rayleigh statistic occurs. These results have been confirmed [1], using a water-based gelatin phantom. The phantom contains plastic scatterers with $100 \mu \mathrm{m}$, and the concentration of gelatin particles was 38 within a $-6 \mathrm{~dB}$ resolution cell of a $2.25 \mathrm{MHz}, 13 \mathrm{~mm}$ transducer.

The ultrasound speckle (which is analogous to speckle in coherent electromagnetic waves) results from the coherent accumulation of random scatterings from the resolution cell as it is scanned through the tissue. The accumulation can be viewed geometrically as a random walk of component phasors. When the number of scatters within one resolution cell is large, and the phases of the scattered waves are distributed uniformly between 0 and $2 \pi$, the magnitude of the phasor that results from the addition of the randomly walking components has a Rayleigh pdf. This is due to the fact that the real and imaginary parts of the phasors have Gaussian density functions. In our simulations, we used a Rayleigh distribution to generate the backscattered signal

$$
p\left(x, \lambda_{2}\right)=\frac{x}{\lambda_{2}^{2}} \exp \left(-2 \frac{x^{2}}{\lambda_{2}^{2}}\right), \quad x=0,1,2, \ldots,
$$

where $x$ is the time distribution of irregular scatterers, and $\lambda_{2}$ is the parameter of Rayleigh distribution (related to the width of the Rayleigh PDF). This parameter is related to the mean time of irregular scatterers $\bar{x}=\mu_{\mathrm{ir}}$ by $\mu_{\mathrm{ir}}=$ $\sqrt{\frac{\pi}{2}} \lambda_{2}$. The time interval between two adjacent irregular scatterers $\Delta \tau_{2}=\tau_{2, i+1}-\tau_{2, i}$ is $\sqrt{\frac{2}{\pi}} x$. In the spatial domain, the MSS $\mu_{\mathrm{d}}$ is $\mu c / 2$ [8], where $c$ is the speed of sound in tissue. In this study, the tissue is considered as a linear time-invariant system, hence the backscattered signal $y(t)$, which results from the interaction of the incident ultrasonic pulse with the scattering structure, is expressed as

$$
y(t)=g(t) \otimes h(t)+n(t)
$$

where $g(t)$ is the impulse response of tissue, $\otimes$ denotes the convolution operator, $n(t)$ is the Gaussian noise with zero mean associated with the ultrasound system 
and $h(t)$ is the transducer impulse response, often assumed to have a Gaussian-shaped frequency spectrum, such as

$$
h(t)=\exp (-\beta t) \cos \left(2 \pi f_{0} t\right) u(t) .
$$

In Eq. (1), the second term is considered to be noise, therefore, using Eqs. (1) and (4), the signal-to-noise ratio (SNRs) is defined as the ratio between the variances of the first term and the second term in the equation

$$
S N R s=10 \log _{10} \frac{\sum_{n} y_{\mathrm{r}}(n)^{2}}{\sum_{n} y_{\mathrm{ir}}(n)^{2}},
$$

where $y_{\mathrm{r}}(n)=g_{\mathrm{r}}(n) \otimes h(n)$ is the backscattered signal from the regular structure, and $y_{\text {ir }}(n)=g_{\text {ir }}(n) \otimes h(n)$ is from the irregular structure. Where, $g_{\mathrm{r}}(n), g_{\mathrm{ir}}(n)$ and $h(n)$ represent the discrete time signal of, respectively, $g_{\mathrm{r}}(t), g_{\text {ir }}(t)$ and $h(t)$.

It was shown in $[13,16]$ that the PSD of a perfectly regular tissue backscattered signal exhibits peaks at harmonic frequencies $f=i f_{1}, i=1,2, \ldots$ of a fundamental frequency $f_{1}$. This property justifies the use of an autoregressive model rather than the moving average (AR) or combined AR and MA (ARMA) models. Considering just the first term of Eq. (1), the PSD of backscattered signal can be given as [8]

$$
S_{\mathrm{g}} g(\omega)=\frac{c}{2 \mu_{\mathrm{d}}}\left[1-2 \alpha \cos \left(2 \omega \mu_{\mathrm{d}} / c\right)\right]
$$

where $\alpha$ is a constant. Equation (7) indicates that a periodic oscillation, the period of which determines the MSS, should be observed. However, the PSD of the echo from the semiregular tissue is altered by speckle noise, which is modeled by the second term in Eq. (1). In addition to the speckle noise, the effect of the impulse response of the transducer introduces another perturbation. For these reasons, it is necessary to estimate the PSD of backscattered signals in order to deduce the MSS. Several authors [7-9] have shown the efficiency of an AR model using the second-order statistics for the estimation of backscattered signals. The same model will therefore be considered in the scope of the TFOC method.

\section{CUMULANTS FOR MSS ESTIMATION}

The PSD of backscattereds signal from regular structures presents equidistant peaks, separated by a frequency increment to the structure period (hence, this property justifies the choice of the AR model [17]), which is proportional to MSS. The MSS estimation is based on the estimation of PSD of the backscattered signal. However, all proposed methods for PSD estimation are based on two important hypotheses: stationarity and Gaussianity. These hypothesis leads to the use of only the second-order statistics (autocorrelation) and the amplitude information while ignoring the phase information. Hence, these hypotheses lead to a very restrictive class of ultrasonic signals backscattered from tissues. In the general case, the signals are non-Gaussian and sometimes even cyclostationary (class between stationary and no stationary) [21]. Higher-order statistics known as cumulants and their associated Fourier transforms, known as polyspectra, reveal not only amplitude information, but also phase information. They also automatically null the effects of colored Gaussian measurement noise, whereas correlation-based methods do not. Therefore, the higher-order statistics appears suitable here. An AR model for backscattered signals, excited by non-Gaussian white noise, is proposed in this section. Its parameters are estimated using the third- and the fourth-order cumulants named CUM3-AR and CUM4-AR. Let the backscattered signal $y(n)$ from tissue be modeled by a non-Gaussian AR model of order $P$ as

$$
y(n)=-\sum_{i=1}^{P} a(i) y(n-i)+v(n) .
$$

The input $\nu(n)$ will have a nonsymmetric and non-Gaussian distribution law, and $a(0)=1$. The system given in Eq. (8) is assumed to be stable and is statistically independent of $v(n)$. If the $k$ th-order cumulant of the driving non-Gaussian noise is nonzero, $y(n)$ has also a $k$ th-order cumulant. This cumulant is defined (for $k=3,4$ ) as [22]

$$
\begin{aligned}
C_{y}^{k}\left(\tau_{1}, \tau_{2}, \ldots, \tau_{k-1}\right)= & E\left[y(t) y\left(t+\tau_{1}\right) \ldots y\left(t+\tau_{k-1}\right)\right] \\
& -E\left[f(t) f\left(t+\tau_{1}\right) \ldots f\left(t+\tau_{k-1}\right)\right]
\end{aligned}
$$

where $E[\cdot]$ denotes the statistical expectation with respect to $t$, and $f(t)$ is a Gaussian process with zero mean. Both $y(n)$ and $f(n)$ have the same auto-covariance.

The second-, third-, and fourth-order cumulants, for a zero mean variable, are given by [22]

$$
\begin{aligned}
C_{2}^{y}(m)= & E[y(n) \cdot y(n+m)], \\
C_{3}^{y}\left(m_{1}, m_{2}\right)= & E\left[y(n) \cdot y\left(n+m_{1}\right) \cdot y\left(n+m_{2}\right)\right], \\
C_{4}^{y}\left(m_{1}, m_{2}, m_{3}\right)= & E\left[y(n) \cdot y\left(n+m_{1}\right) \cdot y\left(n+m_{2}\right) \cdot y\left(n+m_{3}\right)\right] \\
& -C_{2}^{y}\left(m_{1}\right) C_{2}^{y}\left(m_{2}-m_{3}\right) \\
& -C_{2}^{y}\left(m_{2}\right) C_{2}^{y}\left(m_{3}-m_{1}\right) \\
& -C_{2}^{y}\left(m_{3}\right) C_{2}^{y}\left(m_{1}-m_{2}\right) .
\end{aligned}
$$

As previously noted, the AR coefficients of a backscattered signal PSD may be estimated from the following correlation-based normal equations [22]:

$$
\sum_{k=0}^{P} a(k) C_{2}^{y}(k-m)=0, \quad m>0 .
$$


The AR coefficients can also be determined using the third- and the fourth-order cumulants. Hence, using Eqs. (4) and (10), the relation linking the AR model parameters and the cumulants is (the third-order cumulant) given as [23]

$$
\sum_{k=0}^{P} a(k) C_{y}^{3}(k-m, k-n)=\gamma \delta(m, n),
$$

where $\gamma=E\left[v^{2}(n)\right]$.
Once the 3rd-order cumulant is computed, the model parameters $a(k)$ can be estimated by solving Eq. (13) along lines $m=n=0,1, \ldots, P$. The diagonal slice of the thirdorder cumulant sequences is given by the following matrix equation [23]:

$$
J A=B,
$$

where

$$
\begin{aligned}
& J=\left[\begin{array}{rrrr}
C_{3}^{y}(0,0) & C_{3}^{y}(1,1) & \ldots & C_{3}^{y}(P, P) \\
C_{3}^{y}(-1,-1) & C_{3}^{y}(0,0) & \ldots & C_{3}^{y}(P-1, P-1) \\
\cdot & & \ldots & \\
\cdot & & \ldots & \cdot \\
\cdot & \cdot & \ldots & \cdot \\
C_{3}^{y}(-P,-P) & C_{3}^{y}(-P+1,-P+1) & \ldots & C_{3}^{y}(0,0)
\end{array}\right], \\
& A=[1 a(1) \ldots a(P)]^{\mathrm{T}} \text {, }
\end{aligned}
$$

and

$$
B=[\gamma 0 \ldots 0]^{\mathrm{T}} .
$$

The correlation-based method is one way to solve these equtions with $P$ parameters. The second way is that, when we have more equations then unknown parameters, whereby the resulting system (known as higher-order YuleWalker equtions) is overdetermined, the least-squares or singular value decomposition (SVD) is used.

The PSD of the backscattered signal may be estimated from the parameters determined using the fourth-order cumulants. The process to follow is then the same as, to that described above, with identical equations $\left(C_{3}^{y}\right.$ being simply replaced with $C_{4}^{y}$ ). The estimated parametric PSD is smooth, which helps to better localize the amplitude pics and thus estimate the frequency interval. The next section deals with the simulation of the proposed method (CUM3AR and CUM4-AR) and its comparison with that based on second-order statistics (PSD-AR).

\section{RESULTS AND DISCUSSION}

The aim of this part is to demonstrate the efficiency of the TFOC-based method in estimating the MSS. For the simulation, an elaborate (and hopefully realistic) model is used. Monte-Carlo simulations for the three methods (CUM3-AR, CUM4-AR and PSD-AR) are run in order to study their robustness. Using the tissue model described by Eqs. (1) and (4), a large number of data sets (100 realisations) is generated for each combination of parameter values. For each realisation, the MSS is determined from its PSD, which is estimated using CUM3-AR, CUM4AR or PSD-AR. For each set, the mean and the standard deviation of the MSS are then computed and compared.
Table 1 Simulation parameters.

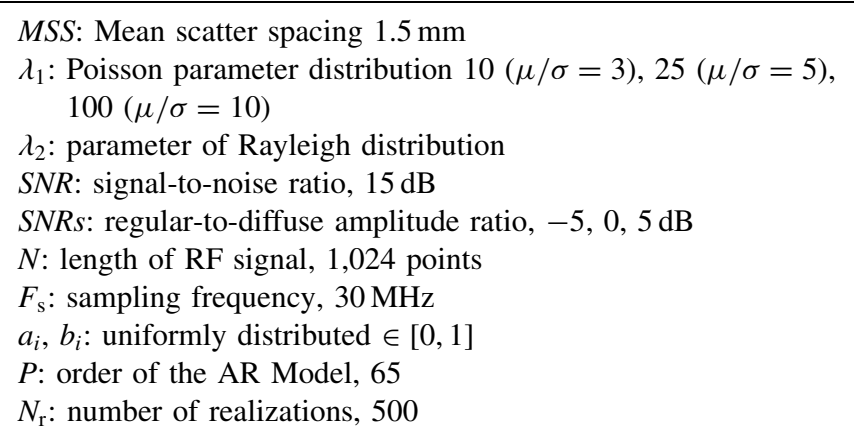

The true MSS $\mu$ is chosen to be equal to $1.5 \mathrm{~mm}$, with distance approximately equal to the size of a liver nodule. The standard deviation of the semiregular spacing $\sigma$ is first varied and the regular spacing to diffuse the amplitude ratio SNRs is kept constant.

According to reference [9], the AR method gives good results if the data window size is greater than $4 \mathrm{~mm}$. Therefore, in our simulation, the data window size is taken to be $25 \mathrm{~mm}$ (window time is $34 \mu \mathrm{s}, \sim 17$ semiregular scatterers by window), corresponding to sampling frequency $F_{\mathrm{s}}=30$ MHz. The simulation parameters are given in Table 1. Our simulation is based on the HOSA toolbox MATLAB [24].

Figure 1(a), 1(b) and 1(c) shows respectively the RF simulated signals of the regular structure, of the irregular structure and the backscattered signal with $S N R s=0 \mathrm{~dB}$ and $S N R=15 \mathrm{~dB}$. Figures 2, 3 and 4 show results of CUM3-AR and PSD-AR for three values of $\sigma$ with SNR value of $0 \mathrm{~dB}$. The corresponding performances for 3 of setresults is shown in Table 2. The black solid lines and dashed lines corresponding to $\mu \pm \sigma$. 

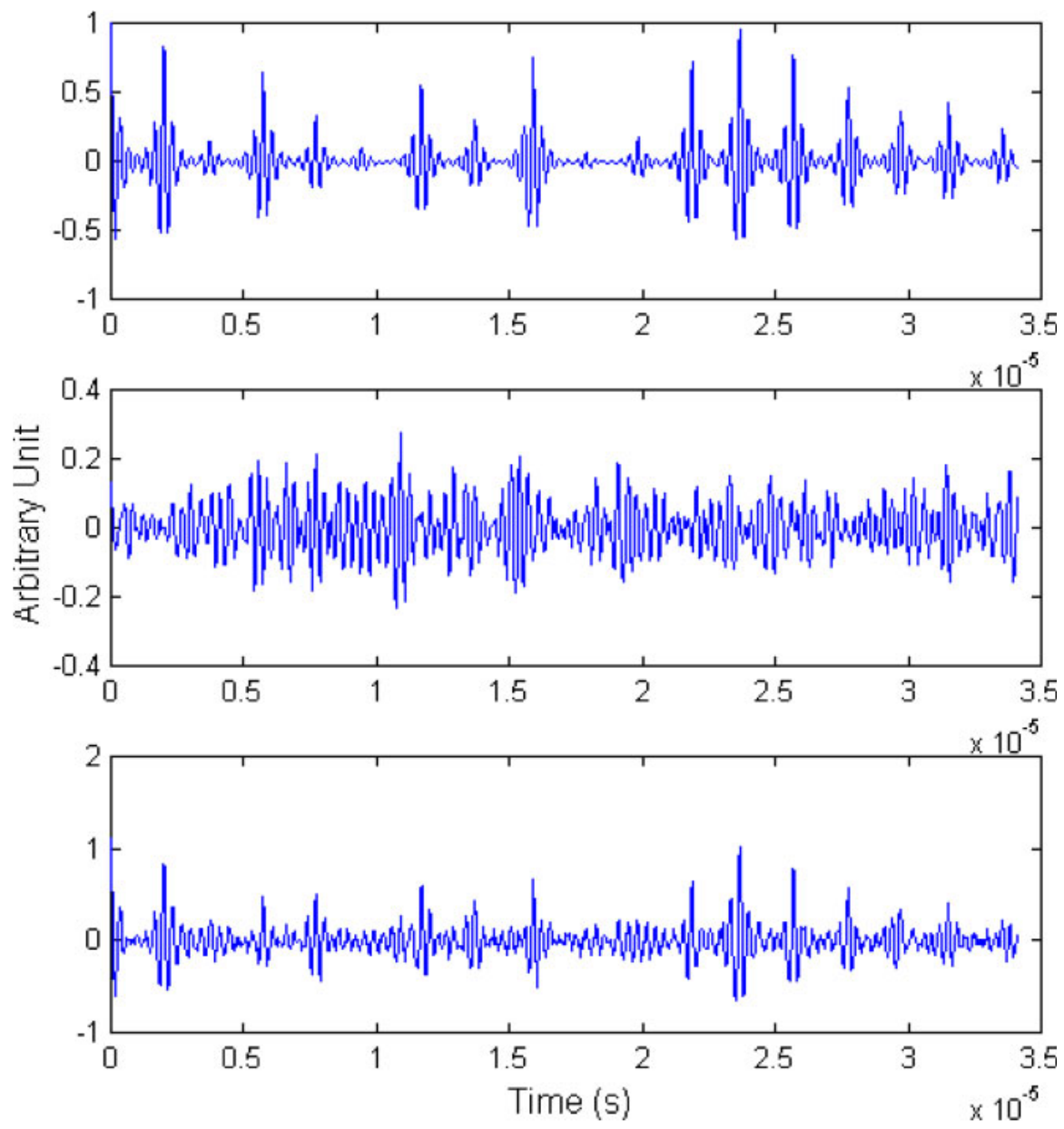

Fig. 1 Simulated signals: a) RF from regular structure, b) RF from irregular structure, c) backscattered signal with $S N R s=0 \mathrm{~dB}$ and $S N R=15 \mathrm{~dB}$.

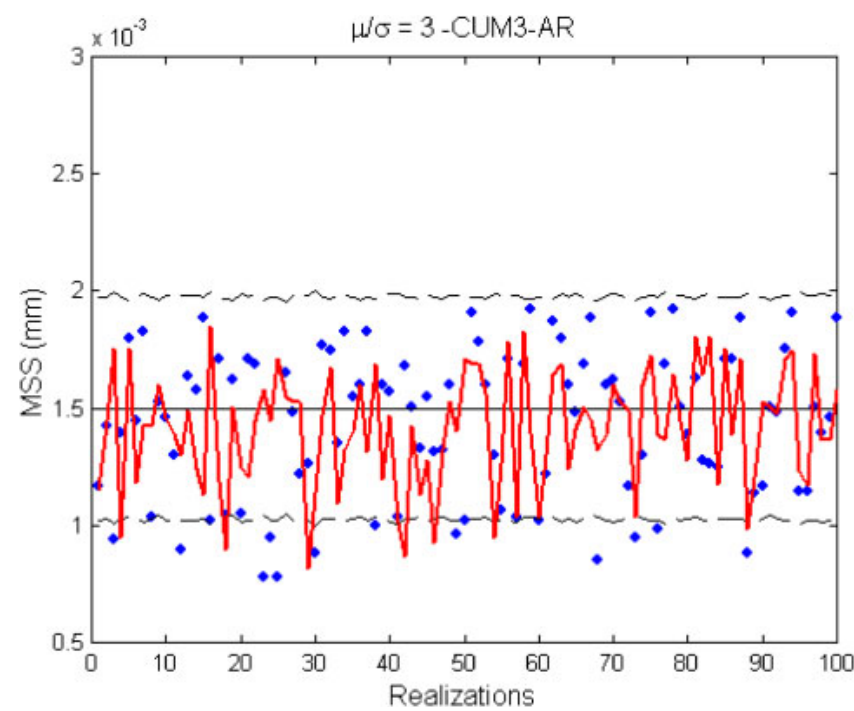

Fig. 2100 realizations of MSS for $\mu / \sigma=3$ and $S N R s=0 \mathrm{~dB}$. CUM3-AR: solid lines, PSD-AR: dots.

The results obtained with CUM4-AR are added even though the results of CUM4-AR are not shown. It is seen that CUM3-AR and CUM4-AR perform better than PSD$\mathrm{AR}$, which is much more sensitive to variations of the

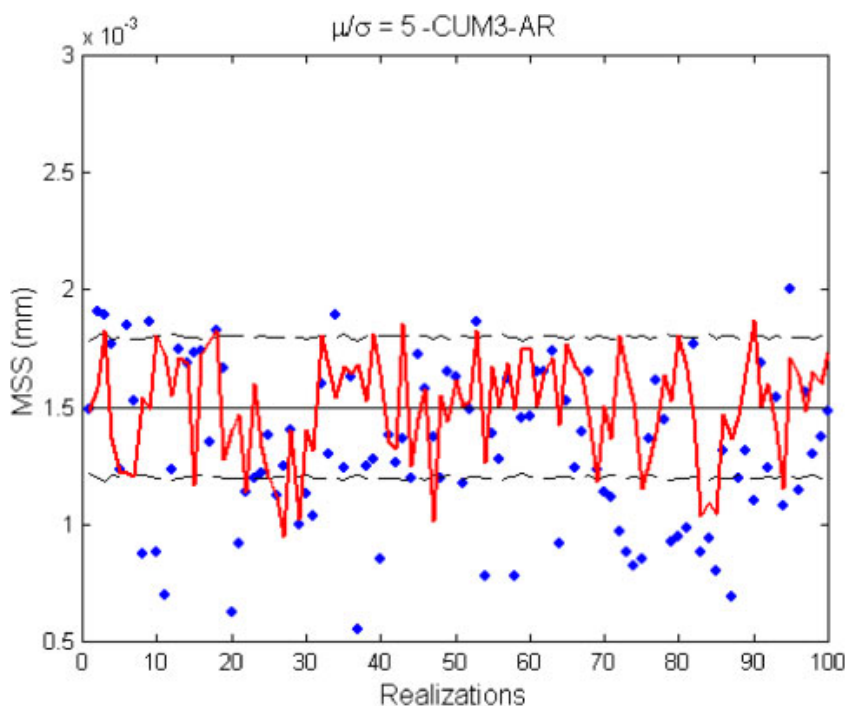

Fig. 3100 realizations of MSS for $\mu / \sigma=5$ and $S N R s=0 \mathrm{~dB}$. CUM3-AR: solid lines, PSD-AR: dots.

standard deviation $\sigma$. For CUM4-AR, the mean value falls closer to the true value and the standard deviation is lower. CUM3-AR also appears to be better than PSD-AR with low variance, giving a good mean value estimate, but a standard 


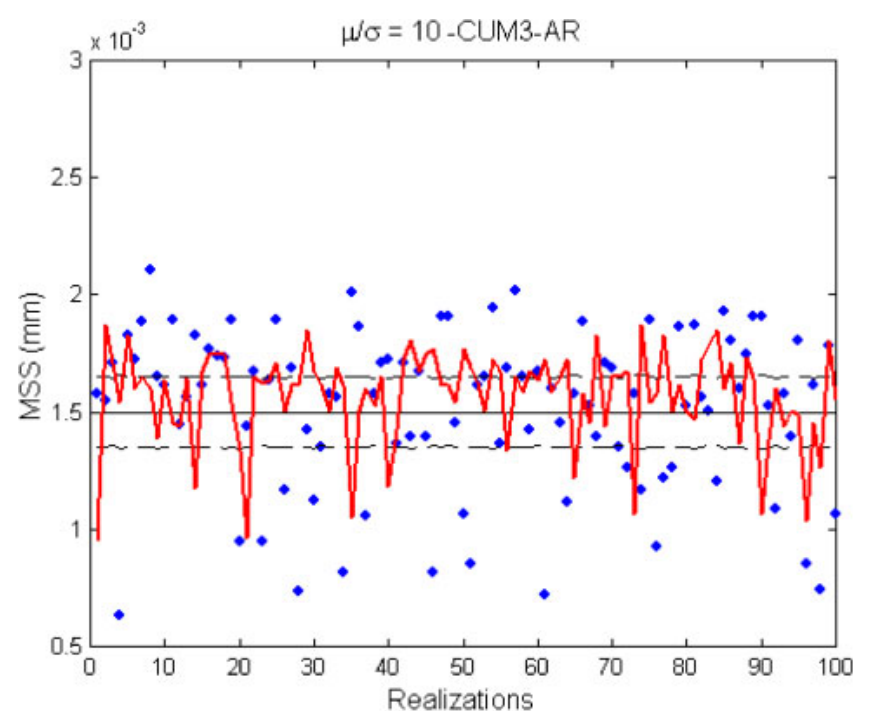

Fig. 4100 realizations of MSS for $\mu / \sigma=10$ and $S N R s=0 \mathrm{~dB}$. CUM3-AR: solid lines, PSD-AR: dots.

Table 2 MSS values (mm) for $S N R s=0 \mathrm{~dB}$.

\begin{tabular}{cccc}
\hline$\mu / \sigma$ & Method & Mean & Variance \\
\hline \multirow{3}{*}{10} & AR & 1.47 & $(0.35)^{2}$ \\
& CUM3-AR & 1.57 & $(0.21)^{2}$ \\
& CUM4-AR & 1.44 & $(0.35)^{2}$ \\
\hline \multirow{3}{*}{5} & AR & 1.37 & $(0.34)^{2}$ \\
& CUM3-AR & 1.49 & $(0.22)^{2}$ \\
& CUM4-AR & 1.50 & $(0.26)^{2}$ \\
\hline \multirow{3}{*}{3} & AR & 1.44 & $(0.30)^{2}$ \\
& CUM3-AR & 1.42 & $(0.24)^{2}$ \\
& CUM4-AR & 1.50 & $(0.26)^{2}$ \\
\hline
\end{tabular}

deviation of the same order (much higher than with CUM3AR). CUM3-AR may therefore be preferred, except when the backscattered signal has a symmetric distribution (CUM4-AR then being a better, natural, choice).

Figures 4, 5 and 6 show results of CUM3-AR and PSDAR for various regular-to-diffuse amplitude ratios, the standard deviation of the semiregular spacing being kept constant $(\mu / \sigma=10)$. The results are summarized in Table 3. It must be noted that CUM3-AR and CUM4-AR give similar results for fixed and variable speckles and yield consistent estimators for MSS, even in the extreme case. Moreover, the performance may be further enhanced by taking a higher number of realizations into account. The CUM4-AR is preferable if the backscattered signal has a symmetric distribution. One point could be a weakness of the parametric-cumulant-based method (even though the fact that is time-consuming is another point but it is not an issue in these applications) which is the stability of our AR model. During the simulation and before estimating the PSD using AR parameters, we check if all poles are inside the unit circle. We noticed that this condition is always verified.

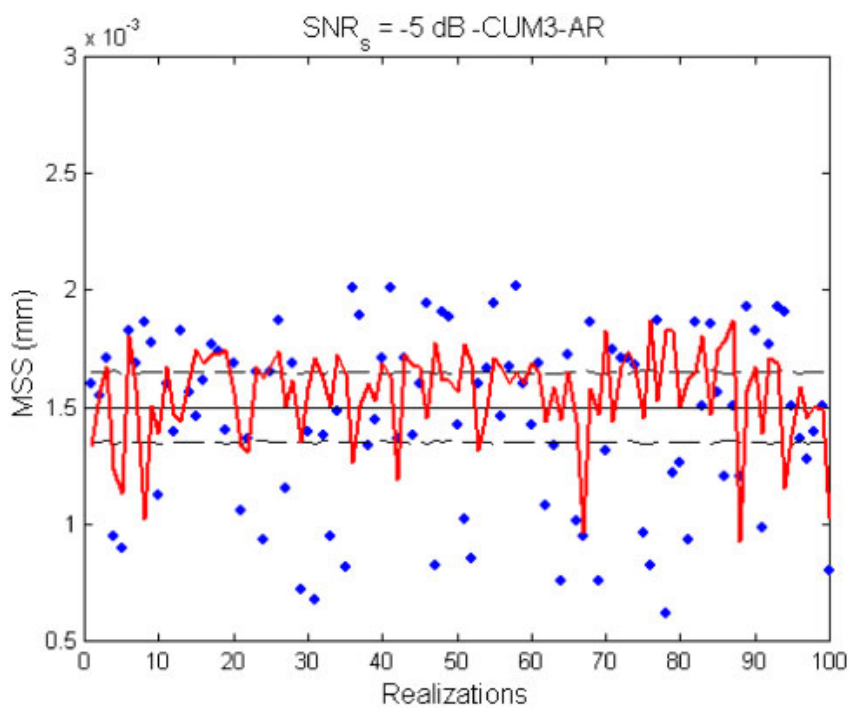

Fig. 5100 realizations of MSS for $\mu / \sigma=10$ and $S N R s=-5 \mathrm{~dB}$. CUM3-AR: solid lines, PSD-AR: dots.

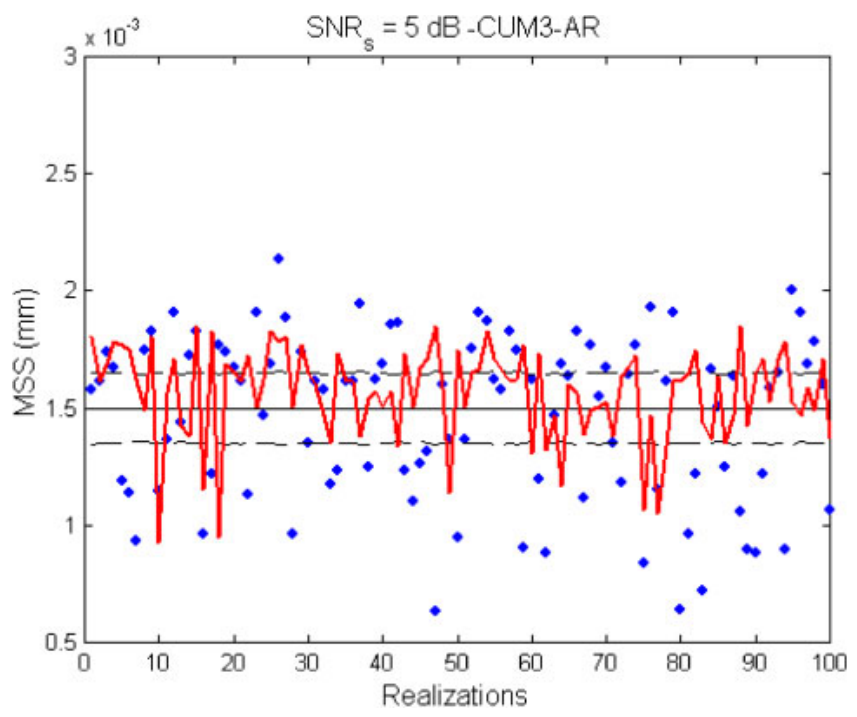

Fig. 6100 realizations of MSS for $\mu / \sigma=10$ and $S N R s=5 \mathrm{~dB}$. CUM3-AR: solid lines, PSD-AR: dots.

Table 3 MSS values (mm) for $\mu / \sigma=100$.

\begin{tabular}{cccc}
\hline SNRs $(\mathrm{dB})$ & Method & Mean & Variance \\
\hline \multirow{3}{*}{-5} & AR & 1.44 & $(0.34)^{2}$ \\
& CUM3-AR & 1.56 & $(0.21)^{2}$ \\
& CUM4-AR & 1.50 & $(0.26)^{2}$ \\
\hline \multirow{2}{*}{0} & AR & 1.47 & $(0.35)^{2}$ \\
& CUM3-AR & 1.57 & $(0.21)^{2}$ \\
& CUM4-AR & 1.45 & $(0.28)^{2}$ \\
\hline \multirow{2}{*}{5} & AR & 1.44 & $(0.35)^{2}$ \\
& CUM3-AR & 1.57 & $(0.21)^{2}$ \\
& CUM4-AR & 1.38 & $(0.21)^{2}$ \\
\hline
\end{tabular}




\section{CONCLUSION}

In this paper we proposed a new method, called TFOC, for MSS estimation using the third-order and the fourthorder cumulants of a parametric AR model for ultrasonic signals backscattered from human tissue. This method was compared with PSD of a parametric AR model. The comparison was based on the performance of each method upon varying the standard deviation of the MSS $\sigma$ and the semiregular-to-irregular signal ratio, which is considered to be a parameter qualifying the homogeneity of the tissue. A set of simulations results (obtained for 100 realizations in each case) showed the good performance of the TFOC method.

\section{REFERENCES}

[1] R. F. Wagner, S. W. Smith, J. M. Sandrik and H. Lopez, "Statistics of speckle in ultrasound B-scans," IEEE Trans. Sonics Ultrason., 30, 156-163 (1983).

[2] C. B. Burckhardt, "Speckle in ultraound B-mode scans," IEEE Trans. Sonics Ultrason., 25, 1-6 (1978).

[3] L. L. Fellingham and G. Sommer, "Ultrasonic characterization of tissue structure in the in vivo human liver and spleen," IEEE Trans. Sonics Ultrason., 31, 418-428 (1984).

[4] L. Landini and L. Verrazzani, "Spectral characterization of tissues microstructure by ultrasounds: A stochastic approach," IEEE Trans. Ultrason. Ferroelectr. Freq. Control, 32, 448-456 (1990).

[5] G. Georgiou and F. S. Cohen, "Statistical characterization of diffuse scattering in ultrasound images," IEEE Trans. Ultrason. Ferroelectr. Freq. Control, 45, 57-64 (1998).

[6] T. Varghese and K. D. Donohue, "Mean scatterer spacing estimates with spectral correlation," J. Acoust. Soc. Am., 96, 3504-3515 (1994).

[7] C. Simon, R. Seip and E. S. Ebbini, "Estimation of mean scatterer spacing based on autoregressive spectral analysis of prefiltered echo data," Proc. Ultrason. Symp., 2, 1153-115 (1995).

[8] C. Simon, J. Shen, R. Seip and E. S. Ebbini, "A robust and computationally efficient algorithm for mean scatterer spacing estimation," IEEE Trans. Ultrason Ferroelectr. Freq. Control, 44, 882-894 (1997).

[9] A. W. Keith, R. F. Wagner, M. F. Insana and J. H. Timothy, "Application of autoregressive spectral analysis to cepstral estimation of mean scatterer spacing," IEEE Trans. Ultrason. Ferroelectr. Freq. Control, 40, 50-58 (1993).

[10] M. F. Insana, T. H. Hall and L. T. Cook, "Backscatter coefficient estimation using array transducers," IEEE Trans. Ultrason. Ferroelectr. Freq. Control, 41, 714-723 (1994).

[11] G. E. Sleefe and P. P. Lele, "Tissue characterization based on scatterer number density estimation," Ultrasound Med. Biol., 14, 749-757 (1988).

[12] C. Simon, "A study on mean scatterers spacing estimation techniques," Ph. D. Dissertation, Department of Engineering and Computer Science, The University of Michigan, pp. 481092122, USA (1996).

[13] T. Varghese and K. D. Donohue, "Estimating mean scatterer spacing with the frequency-smoothed spectral autocorrelation function," IEEE Trans. Ultrason. Ferroelectr. Freq. Control, 42, 451-463 (1995).
[14] C. B. Machado, "Characterization of in vitro healthy and pathological human liver tissue periodicity using backscattered ultrasound signals," Ultrasound Med. Biol., 32, 649-657 (2006).

[15] A. V. Oppenheim and R. W. Schafer, Digital Signal Processing (Prentice-Hall, Englewood Cliffs, New Jersey, 1975).

[16] S. L. Marple, Digital Spectral Analysis with Applications (Prentice-Hall, Englewood Cliffs, New Jersey, 1987).

[17] B. Baligand and M. Grozellier, "Analyse spectrale en contrôle non destructif par ultrasons," Rev. Trait. Signal, 3, 35-43 (1986).

[18] A. Guerchaoui, J. C. Balluet and J. L. Lacoume, "Etude comparative des principales méthodes de déconvolution sur des données de type sismique," Rev. Trait. Signal, 6, 187-203 (1989).

[19] U. R. Abeyrantne, A. P. Petropulu and J. M. Reid, "On modeling the tissue response from ultrasonic B-scan images," IEEE Trans. Med. Imaging, 15, 479-490 (1996).

[20] A. Fort, C. Manfredi and S. Rocchi, "Recursive autoregressive spectral maps for ocular pathology detection," Ultrasound Med. Biol., 23, 391-403 (1997).

[21] J. L. Lacoume, P. O. Amblard and P. Comon, Statistiques d'ordre suprieur pour le traitement du signal (Mason, Paris, 1997).

[22] J. M. Mendel, "Tutorial on high-order statistics (spectra) in signal processing and system theory: Theoretical results and some applications," Proc. IEEE, 79, 278-305 (1991).

[23] J. M. Mendel, M. R. Raghuveer and C. L. Nikias, "Bispectrum estimation via AR modelling," Signal Process., 10, 35-40 (1986).

[24] A. Swami, J. M. Mendel and C. L. Nikias, Higher-order Spectral Analysis Toolbox Users Guide (2001).

Abdelhafid Kaddour was born in Tlemcen, Algeria. He received his Dipl. El. Ing. degree in Electronics, Magister Degree and Doctorat d'Etat in Electroacoustics and Signal Processing from University of Sciences and Technology of Oran, Algeria. Since 1982 he has been a teaching member at the Department of Electronics, USTO. His current research interests include acoustics, electroacoustics, signal processing and visualization of acoustic fields.

Bachir Bouhadef received his degree of "Ingenieur d'Etat" in electronics in 2001 and M.S degree in signal processing 2004 from University of Science and Technology of Oran (USTO). In 2005, he joined the physics department of Pisa university, where he received his Ph.D. degree in applied physics in 2007. From Jan. 2008 to Dec. 2008, he was a Postdoctoral Research Fellow at the same university. From Jan. 2009 to May 2009, he was at University of Sapienza, Physics Department, Rome.

Mohamed Faouzi Belbachir was born in Oran, Algeria. He received his Dipl. El. Ing. degree, Magister Degree and Doctorat d'Etat from the University of Science and Technology of USTO (Oran, Algeria), respectively, in 1976, 1984 and 1991. Since 1981, he has been at the Dept. of Electronics of USTO. He is currently interested in filter design and image processing.

Jean Michel Rouvean was born in 1947, in France. He received his Doctorat in science from University of Paris VI in 1976. Currently, he is a Professor of Electronics at ENSI of mechanical energitic, Valenciennes. His research interests include the application of signal processing for telecommunication, ultrasounds, and radar. 